\title{
Double Trouble: A Synchronous Male Breast Intraductal Papillary Carcinoma with Prostatic Adenocarcinoma
}

\author{
Nurul Hafiez Fijasri ${ }^{1,3}$, Khairunnisa Che Ghazali ${ }^{1,3 *}$, Nurul Hana Mohamad Hassan ${ }^{1,3}$, Wan Zainira Wan Zain ${ }^{1,3}$, \\ Maya Mazuwin Yahya ${ }^{1,3}$, Sharifah Emilia Tuan Sharif ${ }^{2,3}$ and Wan Faiziah Wan Abdul Rahman ${ }^{2,3}$ \\ ${ }^{1}$ Department of Surgery, School of Medical Sciences, University of Science Malaysia, Malaysia
}

${ }^{2}$ Department of Pathology, School of Medical Sciences, University of Science Malaysia, Malaysia

${ }^{3}$ Hospital University of Science Malaysia, Malaysia

Submission: August 18, 2020; Published: August 28, 2020

"Corresponding author: Khairunnisa Che Ghazali, Department of Surgery, Hospital University of Science Malaysia, School of Medical Sciences, University of Science Malaysia, 16150 Kubang Kerian, Kelantan, Malaysia

Abstract

Male breast carcinoma is indeed a rare in predominantly female dominated breast carcinoma. Intraductal papillary carcinoma is an architectural pattern of Ductal Carcinoma in Situ (DCIS) without invasion into the breast stroma. We present a case of 72-year-old male with underlying benign prostatic hyperplasia on 5-alpha reductase inhibitor medication, who had presented with unilateral breast swelling and nipple discharge. While working up for breast carcinoma, there was an incidental finding of prostatic mass from computed tomography. Further assessment from transrectal ultrasound guided (TRUS) biopsy of prostate revealed prostatic adenocarcinoma. Hence, this case highlights the possible etiopathogenic relation between breast and prostate carcinoma.

Abbreviations: DCIS: Ductal Carcinoma in Situ; TRUS: Transrectal Ultrasound Guided; BHP; Benign Prostatic Hyperplasia; USG: Ultrasonography; CT: Computed Tomography; HPE: Histopathological Examination; MBC: Male Breast Carcinoma

\section{Introduction}

Breast cancer in male population is extremely rare, accounting less than $1 \%$ of population [1]. It had been attributed to certain risk factors mainly of BRCA1/2 genetic mutation, increased in estrogen activity and testicular dysfunction [2]. We report a double trouble case when a man recently diagnosed to have intraductal papillary carcinoma of the breast, incidentally, found to have a prostatic adenocarcinoma.

\section{Case Report}

A 72-year-old Malay gentleman with underlying hypertension and benign prostatic hyperplasia $(\mathrm{BPH})$ on tablet Dutasteride, complaint of right breast swelling for two months duration, beneath areolar, remains similar in size, painless, associated with on and off bloody nipple discharge. He had no family history of breast carcinoma, no gynaecomastia, no history of traditional medication or steroids intake. On physical examination of the right breast, there was a $2 \times 2 \mathrm{~cm}$ mass over retroareolar region. The mass was firm in consistency, did not fix to underlying structure, but with serous nipple discharge demonstrated upon squeezing (Figures 1A \& 1B). The contralateral breast was otherwise normal. There were no axillary lymph nodes palpable. Per abdomen and genitalia examination were unremarkable. Digital rectal examination showed enlarged, firm and hard prostate.

Ultrasonography (USG) of the right breast showed a solid cystic lesion within right retroareolar measuring $1.0 \times 1.0 \times 1.8 \mathrm{~cm}$. The solid component measures $0.7 \times 0.9 \mathrm{~cm}$ with minimal vascularity (Figure $2 \mathrm{~A}$ ). Subcentimeter right axillary lymph node with preserved fatty hilum was observed. The needle core biopsy from the lesion revealed papillary ductal carcinoma in situ with intermediate grade (Figure 3A). Estrogen and progesterone receptor were positive, and HER-2 was negative. Computed tomography (CT) of thorax, abdomen and pelvis showed presence of focal enhancing lesion at retroareolar region of right breast measuring $0.8 \times 0.8 \times 1.1 \mathrm{~cm}$. There was no extension to underlying 
pectoralis major muscle and the left breast was normal. There was no lung nodule and no pleural effusion. Liver homogenously enhanced and smooth in outline. The prostate gland was accidentally found enlarged, measuring $9.2 \times 6.4 \times 8.0 \mathrm{~cm}$ with irregular margin and ill-defined heterogeneously enhancing lesion at its anterior aspect. The lesion was seen extending to the base of urinary bladder with poor plane of demarcation between prostate mass and the posteroinferior aspect of urinary bladder. There was poor fat demarcation between the prostate mass with rectum posteriorly (Figure 2B). There was an ill-defined lytic lesion seen in L2 vertebral body, pelvic bone and left proximal femur.
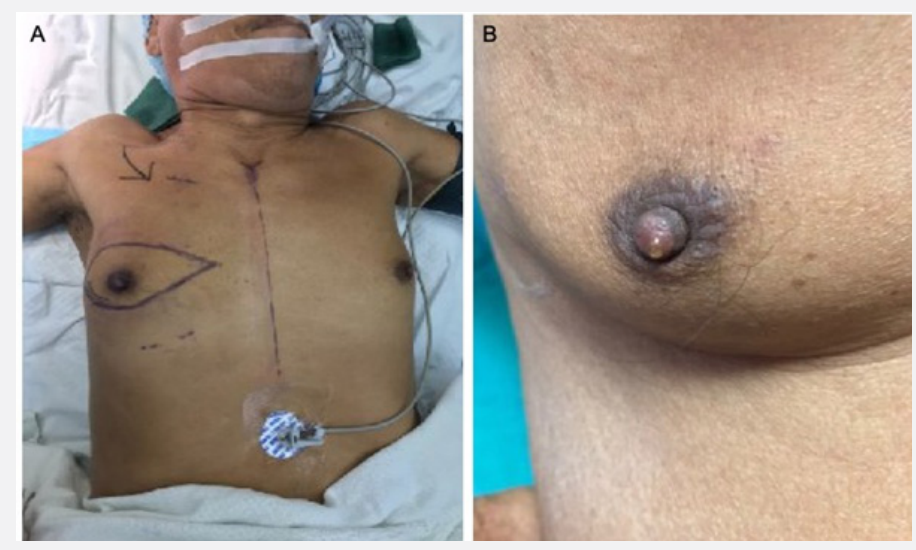

Figure 1: A) Right breast mass with serous nipple discharge. B) Closer view of serous nipple discharge demonstrated.
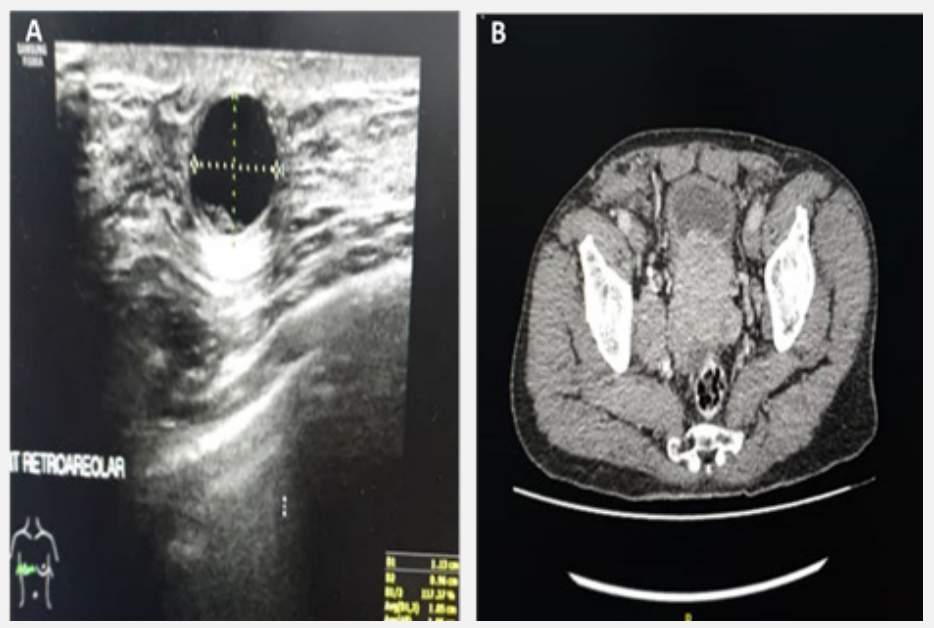

Figure 2: A) USG showed complex cystic mass of the retroareolar right breast. B) CT scan showed ill-defined heterogenous prostatic mass infiltrates to the bladder base.

Following CT finding, the prostatic lesion had been worked up. Serum PSA level was $430.7 \mathrm{ng} / \mathrm{ml}$ and transrectal ultrasound guided (TRUS) biopsy was performed. Histopathological examination showed malignant lesions with extensive cribriform glandular pattern grade 4 within the right prostate and cluster of small, poorly formed gland at the apex of left prostate with absence of basal cell layers. The findings are consistent with prostatic adenocarcinoma Gleason $8(4+4)$. Patient was then undergoing right mastectomy in which post operatively he recovered well and discharge 2 days after operation. Macroscopically, the breast weighing 178 grams with an ellipse of skin (Figures 3C \& D). Serial sectioning showed a whitish firm lesion within retroareolar area (20x20x15mm). Histopathological examination (HPE) confirmed the findings of core biopsy characterised by a cystically dilated central duct, filled with a compact papillary lesion. The lesion was generally dyscohesive with homogenous looking cells, mildly pleomorphic nuclei with coarse chromatin pattern and prominent nucleoli. The peripheral rim of cystically dilated duct is positive for CK5/6 and p63 consistent with myoepithelial cells, however the epithelium and papillary fronds were negative. The remaining ducts and surrounding parenchymal tissue were in keeping with gynaecomastoid hyperplasia. 

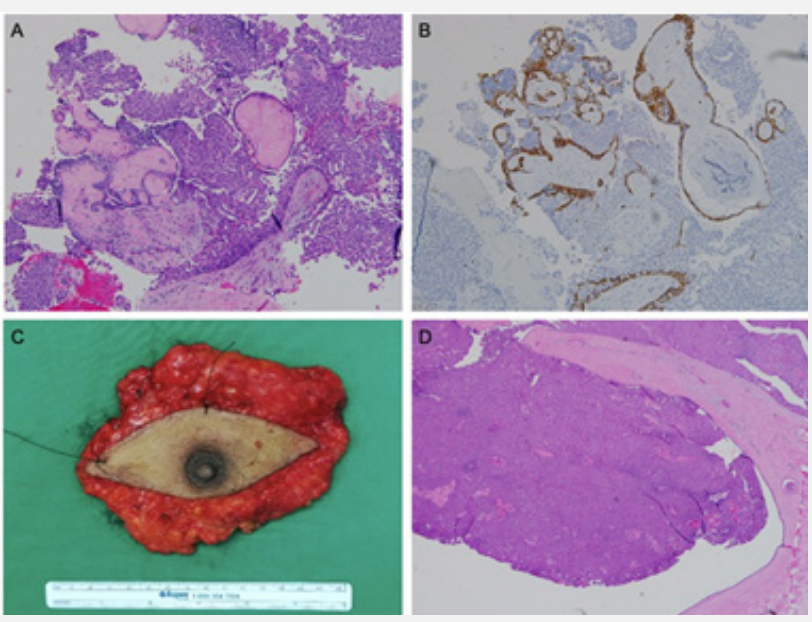

Figure 3: A) Core biopsy from the complex cystic mass of the right breast exhibit cellular tumour cells with papillary core without surrounding stroma indicating of intraductal papillary lesion (H\&E stain, 100x). B) Immunohistochemical (IHC) staining of CK5/6 in core biopsy showed a positivity surrounding the thick fibrous core. However, negative within the tumour cells (IHC stain, 100x). C) Right mastectomy specimen with an ellipse of skin. D) Section from mastectomy confirmed the intraductal lesion characterised by cellular compact tumour within a dilated duct with central thick fibrovascular core (H\&E, 40x).

\section{Discussion}

Breast cancer in male population is rare. Male breast carcinoma (MBC) represents $0.6 \%$ of all breast carcinomas and less than $1 \%$ of all malignancies in men $[1,3]$. In a populationbased study from year 1978-1998 by Giordano et al, the median age at diagnosis for MBC is 67 years compared with 62 years for women with increasing incidence from 0.86 to 1.08 per 100,000 population [1].

Sporadic occurrence of MBC and prostatic carcinoma are even rare. With the advent of the genetic molecular technologies, more knowledge regarding role of genetic become available. Male with BRCA1/2 mutations are at increased risk of cancers of the prostate and breast [4,5]. BRCA2 mutation accounts for $4-20 \%$ of male breast cancer and its carriers have 80 to 100 times higher lifetime risk than in the general population [3,5]. Apart from genetic component, other risk factors include conditions that disproportion the estrogen to androgen ratio. Examples in Klinefelter's syndrome, exogenous estrogen or testosterone use, obesity, abnormalities of testicular condition such as orchitis or epididymitis, testicular ectopia, infertility, finasteride usage or history of prostate cancer treated previously with estrogen, gynaecomastia (though the relationship is still poorly understood) $[3,5]$.

Completion of diagnosis by triple assessment including clinical examination, imaging and histopathological examination. Presenting symptoms are not much different from female breast cancer such as painless mass at the breast associated with nipple discharge and retraction. Lesion usually central and eccentric thus nipple involvement is common $[2,3,6]$. Ultrasonography and mammography are imaging of choice in further assessing lesion in male breast cancer with the latter shows sensitivity and specificity of $92 \%$ and $90 \%$ [5]. In Mathew et al study, pathologic finding of mammogram includes masses that are in high density, spiculated and mostly subareolar or eccentric in location. Mostly shows noncalcified lesion (70\%), while microcalcification found only around $29 \%$ of cases. Masses were mostly irregular in shape while some may in lobular, oval, or round [7]. Ultrasound findings show mass with architectural distortion, with mostly solid or mixed solid cystic component. Most mass are hypoechoic without posterior acoustic phenomenon. Hypervascularity can also demonstrated by power doppler sonography [5,7].

Core biopsy is preferred since it may give value on the invasion of the carcinoma. infiltrating ductal carcinoma account for $85 \%$ making it the most common histological type. In situ ductal carcinoma otherwise may be found in $35-50 \%$ of cases and $5 \%$ of breast cancer in men is pure DCIS with no invasive component. $90 \%$ of tumours are oestrogen- receptor positive and $92-96 \%$ being progesterone-receptor positive [6,8]. Management of male breast cancer depends on the stage during presentation. It can be divided in to surgical, radiotherapy and systemic therapy. Simple mastectomy or modified radical mastectomy has been the method of choice for male breast cancer surgery [8]. In invasive type, axillary dissection is done either by sampling or clearance. Breast reconstruction needs to be planned since up to a third of patient presented at stage III making the primary skin closure a challenge. This can be done by latisimus dorsi or transverse rectus abdominis myocutaneous flap [6].

Indication for radiotherapy is the same with female breast cancer. Examples are extension to the skin, areola, or the pectoralis 
major muscle, inadequate margins, and axillary lymph-node involvement. Besides, multifocality, high grade, high proliferation rate, and peritumoural vascular extensions are factors need to be considered for adjuvant radiotherapy [6,8]. Hormonal treatment is important since most of male breast cancer is hormone receptor positive. In a study by Ribeiro et al shows 5-year survival rate $61 \%$ compared to $44 \%$ of 39 male patients with stage II and III breast cancer treated with tamoxifen compared with controls [6]. Data on adjuvant chemotherapy is well establish due to lack trials however, existing data shows benefit in 5-year surgical rate and overall survival. It can be considered intermediate to high risk primary breast cancer especially hormone receptor negative cases [6]. Treatment for advance disease male breast cancer mainly systemic therapy that are hormonal therapy and palliative chemotherapy. Prognosis depends on stage at presentation and also nodal status. overall 5-year survival are around 40-65\%, while if grouped by stage at presentation, 5-year survival is 75$100 \%$ for stage I disease, $50-80 \%$ for stage II disease, and falling to $30-60 \%$ for stage III disease [6].

\section{Conclusion}

Greater suspicion and further investigation are warranted if men present with breast lumps, nipple discharge or retraction. Approach is the same as female breast cancer and treatment should be tailored to stage at presentation and comorbidity. Support systems should be made available, and further research and trial for breast cancer in men should be conducted at larger scope.

\section{References}

1. Giordano SH, Cohen DS, Buzdar AU, Perkins G, Hortobagyi GN (2004) Breast carcinoma in men: A population-based study. Cancer 101(1): 51-57.

2. Brents M, Hancock J (2019) Novel Insights from Clinical Practice Ductal Carcinoma In situ of the Male Breast.

3. Ruddy KJ, Winer EP (2013) Male breast cancer: risk factors, biology, diagnosis, treatment, and survivorship. Ann Oncol 24 (6): 1434-143.

4. Takuwa H, Tsuji W, Shintaku M, Yotsumoto F (2018) Hormone signaling via androgen receptor affects breast cancer and prostate cancer in a male patient: A case report 18(1): 1282.

5. Sonia Z, Wiem K, Ibtissem A, Salem AB, Salma A, et al. (2017) Male Breast Cancer: Case Studies and Literature Review. Open Access Libr J.

6. Fentiman IS, Fourquet A, Hortobagyi GN (2006) Male breast cancer. Lancet 367(9510): 595-604.

7. Jean Mathew, George H, Perkins, Tanya Stephens, Lavinia P (2008) Primary Breast Cancer in Men: Clinical, Imaging, and Pathologic Findings in 57 Patients Jean. Am J Radiol 191 (6): 1631-1639.

8. Sara Javidiparsijani, Lauren E, Rosen PG (2017) Male Breast Carcinoma: A Clinical and Pathological Review. Int J Surg Pathol 25(3): 200-205.

\begin{tabular}{l} 
Your next submission with Juniper Publishers \\
will reach you the below assets \\
- Quality Editorial service \\
- Swift Peer Review \\
- Reprints availability \\
- E-prints Service \\
- Manuscript Podcast for convenient understanding \\
- Global attainment for your research \\
- Manuscript accessibility in different formats \\
( Pdf, E-pub, Full Text, Audio) \\
- Unceasing customer service \\
Track the below URL for one-step submission \\
https://juniperpublishers.com/online-submission.php \\
\hline
\end{tabular}

